\title{
The role of sagittal pelvic morphology in the development of adult degenerative scoliosis
}

\author{
Steven de Reuver ${ }^{1}[$ ( $)$ Philip P. van der Linden ${ }^{1} \cdot$ Moyo C. Kruyt ${ }^{1} \cdot$ Tom P. C. Schlösser $^{1} \cdot$ René M. Castelein $^{1}$
}

Received: 12 March 2021 / Revised: 15 May 2021 / Accepted: 6 July 2021 / Published online: 22 July 2021

(c) The Author(s) 2021

\begin{abstract}
Purpose Pelvic morphology dictates the alignment and biomechanics of the spine. Recent observations in different types of adolescent idiopathic scoliosis indicate that individual pelvic morphology is related to the spinal levels in which scoliosis develops: primary lumbar adolescent scoliosis is associated with a higher pelvic incidence (PI) than thoracic scoliosis and non-scoliotic controls. We hypothesize that adult degenerative scoliosis (ADS) of the lumbar spine follows the same mechanical principles and is associated with a high PI.

Methods This study used an existing CT-scan database, 101 ADS patients were sex and age matched to 101 controls. The PI was measured by two observers with multi-planar reconstruction, perpendicular to the hip-axis according to a previously validated technique.

Results The PI was $54.1^{\circ} \pm 10.8^{\circ}$ in ADS patients and $47.7^{\circ} \pm 10.8^{\circ}$ in non-scoliotic controls $(p<0.001)$. The median ADS curve apex was the disc L2-3 and median curve length was 4 vertebral levels. The mean supine Cobb angle was $21^{\circ} \pm 8^{\circ}$ (ranged $\left.10^{\circ}-47^{\circ}\right)$. There was no significant correlation between PI and the apex level $(p=0.883)$, the curve length $(p=0.418)$ or the Cobb angle $(p=0.518)$.

Conclusions ADS normally develops de novo in the lumbar spine of patients with a higher PI than controls, similar to primary lumbar adolescent idiopathic scoliosis. This suggests a shared mechanical basis of both deformities. Pelvic morphology dictates spinal sagittal alignment, which determines the segments of the spine that are prone to develop scoliosis.
\end{abstract}

Keywords Adult degenerative scoliosis $\cdot$ Sagittal pelvic morphology $\cdot$ Spino-pelvic alignment $\cdot$ Pelvic incidence $\cdot$ Sex-age matched controls

\section{Introduction}

The unique upright sagittal profile of the human spine and its consequences for human spinal biomechanics provide an important mechanical basis for the development of spinal deformities [1-5]. Spinal alignment and consequent biomechanics are to a large extent determined by the pelvic incidence (PI), first described by Duval-Beaupère et al. [6]. The PI describes position-independent sagittal pelvic morphology and is strongly related to the sagittal spinal configuration, making it ideal for studying and comparing spino-pelvic alignment [7]. Also, the PI is not influenced

René M. Castelein

r.m.castelein@umcutrecht.nl

1 Department of Orthopaedic Surgery, G05.228, University Medical Center Utrecht, P.O. Box 85500, 3508 GA Utrecht, The Netherlands by potential spinal deformity and is therefore a pre-existent parameter, facilitating the study of cause-and-effect relationships. The PI can be measured on conventional lateral spinal radiographs that include both femoral heads. Recent studies show that the inaccuracy of the projection plane accounts for a variability of $3^{\circ}-6^{\circ}$ in PI when measured on conventional radiographs, compared to $0.8^{\circ}$ when measured on $3 \mathrm{D}$ computed tomography (CT) images [8-11]. CT measurements, however, are acquired in a horizontal position and will never become popular for general assessment due to the high radiation burden, but existing databases of CT-scans obtained for indications such as polytrauma screening or malignancies, can be used for study purposes.

Earlier studies observed a higher PI in primary lumbar adolescent idiopathic scoliosis patients compared to primary thoracic curve types and to non-scoliotic controls [7, 12-14]. In adolescents, pelvic morphology dictates the spinal alignment, where a higher PI corresponds with a more 
pronounced curvature of the spinal profile in the sagittal plane, including a steeper dorsally inclined segment of the thoracolumbar spine [15]. This dorsally inclined segment appears to be of causal importance. A recently published study demonstrated that the inclination and magnitude of this dorsally inclined area in the years before the onset of scoliosis, differs between those that will and will not develop scoliosis [16].

Also in adults, it has been shown that the sagittal profile plays a role in the development of adult degenerative scoliosis (ADS) of the lumbar spine, suggesting the importance of spino-pelvic alignment also in this type of lumbar scoliosis $[2,17,18]$. We hypothesize a similar mechanical basis of both adolescent and adult de novo lumbar scoliosis. Plain radiography studies have already suggested the relationship of a slightly higher PI in ADS patients compared to either patients with milder ADS, poorly defined controls and/or a literature standard PI value [19-21]. The purpose of this study was to provide a thorough determination of the relationship between PI and ADS, by measuring the PI in an existing CT-scan database and compare ADS patients to a sex and age matched control population.

\section{Methods}

\section{Study population}

The Institutional Review Board (IRB) exempted patient informed consent for this retrospective cross-sectional comparative study (IRB number 19/642). All patients aged 40-80 that had received a full body CT-scan between 2011 and 2019 in our clinic and had a diagnosis of ADS with a Cobb angle $>10^{\circ}$ according to the Scoliosis Research Society guidelines were included [22]. Patients were included only if there was no history of spinal surgery, no previous hip arthroplasty, both femoral heads were visible on the CT-scan, no pelvic or vertebral fractures and no other spinal deformities, such as idiopathic scoliosis, were present. From the same hospital, patients aged 40-80 that had received a full-body CT-scan between 2011 and 2019 for indications not related to the spine were used as potential control patients and screened for the same criteria as the ADS patients except the presence of a scoliotic curve with a Cobb angle $>10^{\circ}$. Before further analysis of the CT-scans and blinded for all baseline characteristics except sex and age, every included ADS patient was matched to a control patient of first the same sex, and thereafter of the closest age. Of the ADS patients and sex-age matched controls, basic characteristics of the spine were determined on digitally reconstructed radiographs of the CT-images: supine coronal Cobb angle, curve convexity, apex level and the curve length recorded as the number of vertebral bodies in the curve from Cobb-to-Cobb end vertebrae, following the Scoliosis Research Society guidelines [22].

\section{Three-dimensional pelvic incidence measurement}

In the current study, we used a previously validated, accurate 3D method that uses sagittal plane reconstructions perpendicular to hip-axis $[9,14]$. The PI is measured as the angle between the line from the femoral-heads-axis to the midpoint of the sacral endplate, and the line perpendicular to the sacral endplate (Fig. 1). The supine full body CT-scans of every included ADS patients and sex-age matched control were analysed by multi-planar reconstruction (MPR) to measure the PI (Fig. 2).

\section{Statistical analysis}

The required sample size was calculated based on an earlier study that measured the pelvic incidence with the same method on CT-scans of an asymptomatic control population $\left(47.1^{\circ} \pm 10.0^{\circ}\right)$ [9]. To detect a difference of 5 degrees in PI or more with $80 \%$ power and a two-sided alpha of $5 \%$ in two equally sized groups (sampling ratio of 1), a required sample size of 63 per group was calculated [23]. All descriptive statistics were tested for normality with a Shapiro-Wilk test. For normally distributed parameters the mean, range and standard deviation (SD) were calculated and for not

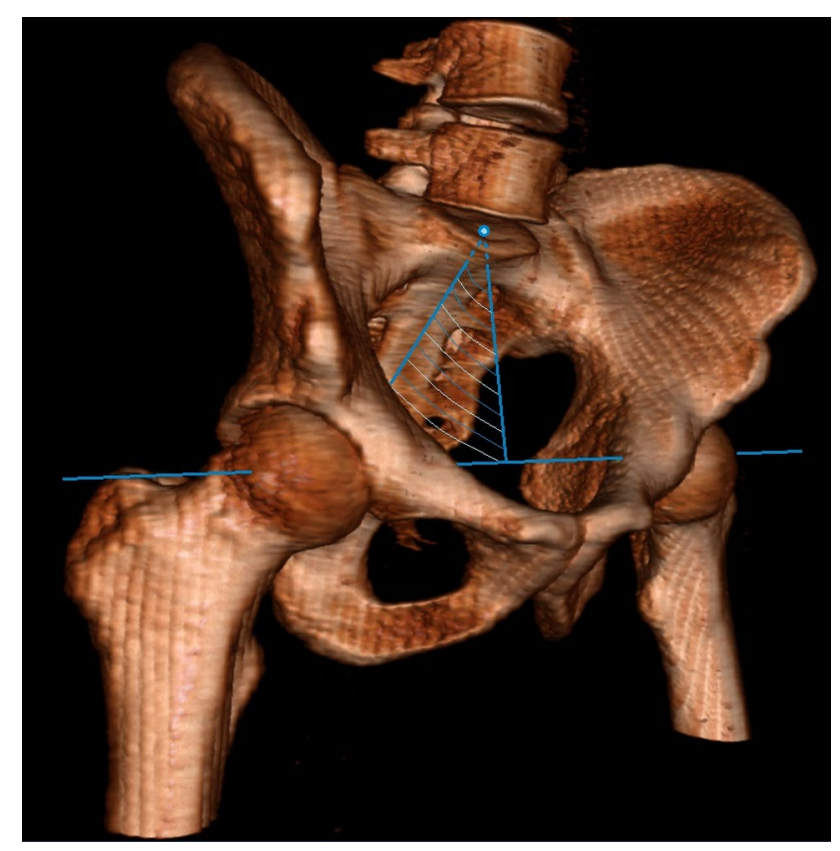

Fig. 1 A three-dimensional (3D) CT reconstruction of the femoral heads, pelvis and lower lumbar spine. The pelvic incidence is shown in the light blue lined plane. PI is defined as the angle between the line from the femoral-heads-axis to the mid-point of the sacral endplate, and the line perpendicular to the sacral endplate 


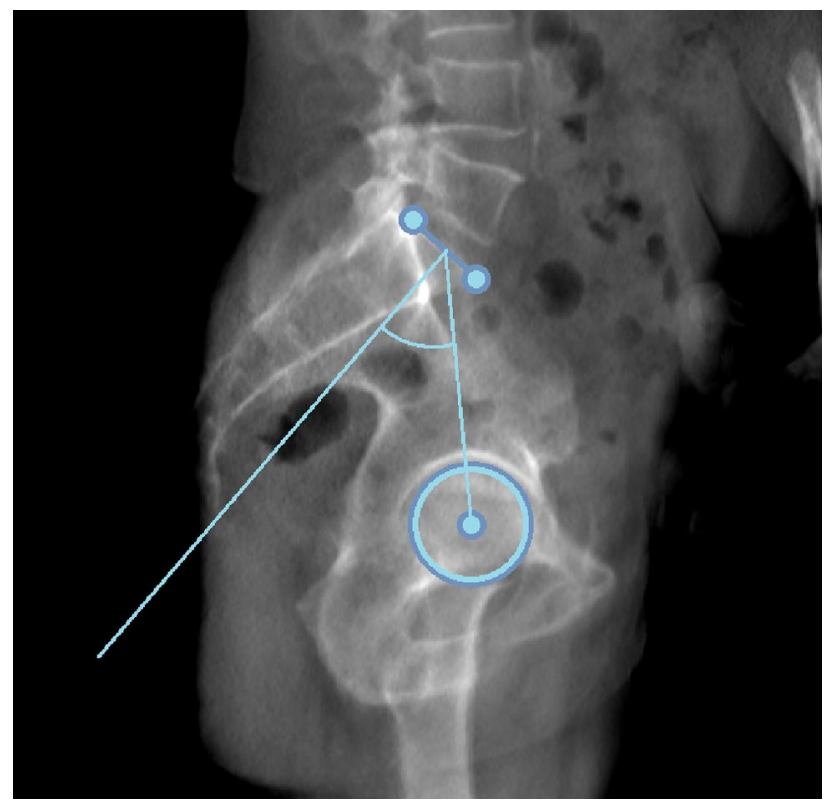

Fig. 2 Full body CT-scans of all included patients were analysed by multi-planar reconstruction (MPR) to get a three-dimensional (3D) image rotatable in all planes. The femoral heads were aligned and encircled by the observer to get the exact axis through the femoral heads. After selecting the sacral endplate, the pelvic incidence was measured as the angle between the line from the femoral-heads-axis to the mid-point of the sacral endplate, and the perpendicular line from the mid-point of the sacral endplate

normally distributed parameters the median and interquartile range (IQR) were calculated. Percentages were shown for the categorical variables. The difference in PI between the two groups was analysed with an independent samples $t$ test. A linear regression analysis between age and PI in both ADS patients and sex-age matched controls was performed. For curve characteristics, a Pearson's correlation test between the apex level and curve convexity was performed, also the association between PI and the apex level, curve length and Cobb angle were analysed with an ordinal regression analysis. Statistical analyses were performed with SPSS 25.0 for Windows (IBM, Armonk, NY, USA). The statistical significance level was set at 0.05 .

\section{Results}

A total of 101 ADS patients were sex and age matched to 101 controls. The median age was 69 in ADS patients and 68 in the matched controls, $57 \%$ were female (Table 1). In ADS patients, the median apex level was the disc L2-3, the mean coronal Cobb angle was $21^{\circ} \pm 8^{\circ}$ and ranged from $10^{\circ}$ to $47^{\circ}$ (Table 1). Furthermore, all ADS curves had their apex in the lumbar area, in concurrence with earlier observations of de novo ADS, and $56 \%$ of these primary lumbar curves
Table 1 Demographics and results

\begin{tabular}{lll}
\hline & ADS & Control \\
\hline$N$ & 101 & 101 \\
Median year of age & 69 & 68 \\
Female & $57 \%$ & $57 \%$ \\
Mean Cobb angle $( \pm \mathrm{SD})$ & $21^{\circ} \pm 8^{\circ}$ & \\
Range & $10^{\circ}$ to $47^{\circ}$ & \\
Convexity of lumbar curve & $56 \%$ left-sided & \\
Median apex & $\mathrm{L} 2-3$ & \\
IQR & $\mathrm{L} 2$ to L3 & \\
Median curve length (included & 4 & \\
$\quad$ number of vertebrae) & & \\
IQR & 3 to 5 & $47.7^{\circ} \pm 10.8^{\circ}$ \\
Pelvic incidence* & $54.1^{\circ} \pm 10.8^{\circ}$ &
\end{tabular}

Demographics and results: A total of 101 adult degenerative scoliosis (ADS) patients were compared to 101 sex and age matched control patients. L2-3: the intervertebral disc between vertebral body L2 and L3. IQR: interquartile range. *: statistically significant difference, $p<0.001$

were left convex (Table 1) [24]. A higher lumbar apex level correlated significantly with a right convex curve $(R=0.282$; $p=0.004)$.

The PI was $54.1^{\circ} \pm 10.8^{\circ}$ in $\operatorname{ADS}$ patients and $47.7^{\circ} \pm 10.8^{\circ}$ in sex-age matched controls $(p<0.001$; Table 1). Furthermore, there was a weak and slightly significant correlation between age and PI in both the ADS group $(R=0.225 ; p=0.024)$ and the sex-age matched controls $(R=0.197 ; p=0.048 ;$ Fig. 3$)$. There was no significant correlation between PI and the specific level of the apex $(p=0.883)$, the length of the scoliotic curve $(p=0.418)$ or the Cobb angle $(p=0.518)$.

\section{Discussion}

The mechanical basis of scoliosis is becoming more and more understood. The human spine has a unique sagittal profile, that renders certain spinal segments prone to decompensate into a scoliotic deformity $[2,4,5]$. How, why and when this decompensation starts is still matter of continuing investigations, however, not yet fully understood mechanical properties of the maturing as well as the degenerating disc as the primary passive stabilizer of the spine likely play a role in the pathogenesis of scoliosis $[4,25,26]$. Pelvic morphology influences the sagittal spinal profile, e.g. a higher PI typically results in a more pronounced sagittal spinal curvature and a steeper dorsal inclination of the lumbar spine $[6,7$, 15]. Furthermore, a higher PI has been shown to be related to primary lumbar adolescent idiopathic scoliosis, whereas a lower PI was associated with primary thoracic curves and non-scoliotic controls [7, 12-14]. In de novo adult spinal 

the pelvic incidence and age for all males and females in the adult degenerative scoliosis (ADS) group and sex-age matched control group. Linear regression analysis showed a weak but significant correlation between age and pelvic incidence in both the ADS group $(R=0.225 ; p=0.024)$ and the sex-age matched controls $(R=0.197 ; p=0.048)$, indicated by the dashed lines in figure
Fig. 3 This scatter-plot shows

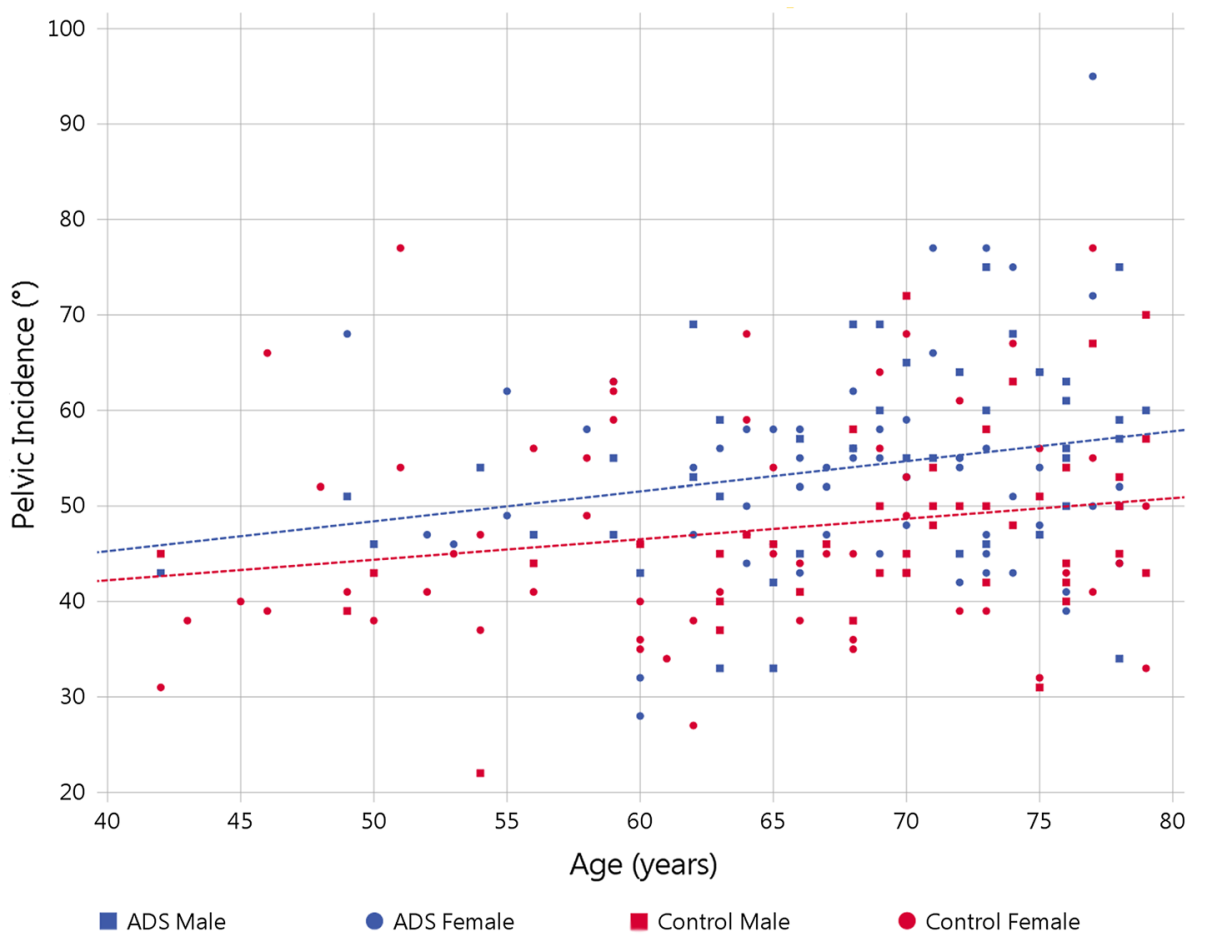

deformities, the curvature normally develops in the lumbar spine during a phase in life when the torsional stiffness of the intervertebral disc decreases [18, 24, 25, 27-29]. If mechanical characteristics, dictated by the sagittal pelvic morphology, play a role in the development of scoliosis, we expect a similar phenomenon of a higher PI in ADS as was observed in primary lumbar adolescent idiopathic scoliosis. So far, three radiographic studies indicated a slightly higher PI in ADS patients compared to either patients with milder ADS, controls and/or a literature standard PI value [19-21].

This CT-based study with sex and age matched controls demonstrated that the PI was indeed higher in ADS than in non-scoliotic controls ( $54^{\circ}$ vs $48^{\circ}$; Table 1$)$. Since PI is not affected by the spinal deformity, this confirms the role of sagittal spino-pelvic alignment in the development of both idiopathic adolescent and adult degenerative de novo scoliosis. Using the same CT-based method for PI measurements, a similar but slightly larger PI difference of $51^{\circ}$ in (thoraco) lumbar adolescent idiopathic scoliosis and $41^{\circ}$ in matched controls was observed [14]. These overall lower PIs compared to the current observations of $54^{\circ}$ in $\mathrm{ADS}$ and $48^{\circ}$ in controls, are explained by the fact that children and adolescents are known to have a lower PI than adults [30-33]. This ageing effect was also observed in the current study (Fig. 3). Furthermore, all ADS curves had their apex in the lumbar area, which is consistent with data from the literature [24, 25, 28] (Table 1). And a higher lumbar apex correlated significantly with a right-convex curve, which is in concurrence with observations that scoliotic curve convexity tends to follow the slight pre-existent rotational pattern present in the non-scoliotic spine [34].

The three main strengths of the current study are: First, the comparison between ADS patients and a sex-age matched control population. Second, the study was powered to detect a difference of 5 degrees in PI or more [9, 23]. And third, the use of 3D CT-scan images and a validated measurements method of the PI, which is more accurate than radiographic measurements [8-11]. Due to the inevitable nature of the CT-scans, made supine in contrast to the upright position during standard full spine radiography, a potential limitation is that some smaller ADS curves may have dropped below the 10-degree threshold in the supine position, and were therefore not included in the ADS group. Furthermore, it is possible that patients with an unknown history of adolescent idiopathic scoliosis with only a lumbar curve (Lenke 5), or similarly for adult idiopathic scoliosis, that developed a degenerative lumbar spine over time, were included in this study as ADS patients. However, the prevalence of this occurrence is likely to be very small compared to the reported ADS prevalence of $30-60 \%$, and therefore the influence on the results is insignificant [25].

The current and earlier studies again confirm the interaction between the sagittal spinal profile as dictated by the PI and the occurrence of scoliosis [1-7]. This strengthens the hypothesis of a causal relationship between sagittal spinal morphology and development of both idiopathic adolescent and adult degenerative scoliosis [7, 12-14]. In this causal mechanism, the individual's PI in itself is not a sufficient 
cause or 'trigger', but a component cause, predisposing patients with a specific spino-pelvic alignment to develop scoliosis in different areas of the spine, dependent of the sagittal profile, during two separate phases of life. In both adolescence and in later adulthood, important changes to spinal biomechanics occur $[4,25,26]$. We propose that the 'trigger' is a relative loss of equilibrium between the passive stabilizers, mainly the intervertebral discs, on the one hand and the spinal loading on the other. During adolescence, an imbalance can occur between rapidly increasing loads on the spine and the mechanical properties of the still maturing disc, where initially a cartilaginous insertion of the annulus fibrosus fibres changes into a bony insertion during puberty, with ossification and fusion of the ring apophysis to the vertebral endplate [35-37]. Similarly at later age, degeneration related loss of torsional stiffness can induce a segmental spinal instability under relatively constant spinal loading, a process potentially sped up by a high PI itself, by dictating a more pronounced lumbar lordosis, increasing local stress on intervertebral discs and therefore uneven degeneration may take place $[18,29,38]$.

However, for both phases in life, the spinal level where this (relative or absolute) loss of mechanical properties of the intervertebral discs will become manifest, appears to be dictated by the sagittal spinal profile. Explained by the Roussouly classification, a higher PI is associated with a more pronounced curvature of the spinal profile in the sagittal plane, including a steeper dorsally inclined segment of the lumbar spine [15]. The resultant dorsal shear forces in these sections are known to decrease rotational stability $[2,17]$. And already before scoliosis onset, this dorsally inclined segment was observed to be different between those that later developed a primary thoracic or a lumbar curve, or did not develop a scoliosis at all [16]. This suggests an important role for sagittal spino-pelvic alignment in a general mechanism of scoliosis development during two separate phases of life, characterized by a change in the balance between mechanical properties of the disc in relation to the spinal loading.

\section{Conclusions}

Adult degenerative scoliosis develops de novo in the lumbar spine of patients with a higher PI than controls, similar to lumbar adolescent idiopathic scoliosis. This suggests a shared mechanical basis of both deformities. It is known that pelvic morphology dictates spinal sagittal alignment, which determines the segments of the spine that are prone to develop scoliosis. We hypothesize that whether and during which phase in life this will occur, depends on the mechanical properties of the passive stabilizers of the spine, predominantly the discs, either during maturation, or during degeneration.

\section{Declaration}

Conflict of interest Steven de Reuver: nothing to declare. Philip van der Linden: nothing to declare. Moyo C. Kruyt: nothing to declare. Tom P.C. Schlösser: nothing to declare. René M. Castelein: Stryker Spine Research Grant. All authors contributed equally to this work by either writing, editing, brainstorming and/or supervising the current manuscript. Supporting data are available upon request.

Open Access This article is licensed under a Creative Commons Attribution 4.0 International License, which permits use, sharing, adaptation, distribution and reproduction in any medium or format, as long as you give appropriate credit to the original author(s) and the source, provide a link to the Creative Commons licence, and indicate if changes were made. The images or other third party material in this article are included in the article's Creative Commons licence, unless indicated otherwise in a credit line to the material. If material is not included in the article's Creative Commons licence and your intended use is not permitted by statutory regulation or exceeds the permitted use, you will need to obtain permission directly from the copyright holder. To view a copy of this licence, visit http://creativecommons.org/licenses/by/4.0/.

\section{References}

1. Marty C, Boisaubert B, Descamps H et al (2002) The sagittal anatomy of the sacrum among young adults, infants, and spondylolisthesis patients. Eur Spine J 11:119-125

2. Castelein RM, van Dieën JH, Smit TH (2005) The role of dorsal shear forces in the pathogenesis of adolescent idiopathic scoliosis-a hypothesis. Med Hypotheses 65:501-508

3. Cheng JC, Castelein RM, Chu WC et al (2015) Adolescent idiopathic scoliosis. Nat Rev Dis Prim 1:15-30

4. Castelein RM, Pasha S, Cheng JC, Dubousset J (2020) Idiopathic scoliosis as a rotatory decompensation of the spine. $\mathrm{J}$ Bone Miner Res 35:1850-1857

5. Pasha S (2019) 3D deformation patterns of s shaped elastic rods as a pathogenesis model for spinal deformity in adolescent idiopathic scoliosis. Sci Rep 9:16485

6. Duval-Beaupère G, Schmidt C, Cosson P (1992) A barycentremetric study of the sagittal shape of spine and pelvis: The conditions required for an economic standing position. Ann Biomed Eng 20:451-462

7. Legaye J, Duval-Beaupère G, Hecquet J, Marty C (1998) Pelvic incidence: A fundamental pelvic parameter for three-dimensional regulation of spinal sagittal curves. Eur Spine J 7:99-103

8. Vaz G, Roussouly P, Berthonnaud E, Dimnet J (2002) Sagittal morphology and equilibrium of pelvis and spine. Eur Spine $\mathrm{J}$ 11:80-87

9. Vrtovec T, Janssen MMA, Pernuš F et al (2012) Analysis of pelvic incidence from 3-dimensional images of a normal population. Spine (Phila Pa 1976) 37:E479-E485

10. Ghostine B, Sauret C, Assi A et al (2017) Influence of patient axial malpositioning on the trueness and precision of pelvic parameters obtained from 3D reconstructions based on biplanar radiographs. Eur Radiol 27:1295-1302 
11. Moon JW, Shinn JK, Ryu D et al (2017) Pelvic incidence can be changed not only by age and sex, but also by posture used during imaging. Korean J Spine 14:77-83

12. Schlösser TPC, Shah SA, Reichard SJ et al (2014) Differences in early sagittal plane alignment between thoracic and lumbar adolescent idiopathic scoliosis. Spine J 14:282-290

13. Pasha S, Aubin C-E, Sangole AP et al (2014) Three-Dimensional Spinopelvic Relative Alignment in Adolescent Idiopathic Scoliosis. Spine (Phila Pa 1976) 39:564-570

14. Brink RC, Vavruch L, Schlösser TPC et al (2019) Three-dimensional pelvic incidence is much higher in (thoraco)lumbar scoliosis than in controls. Eur Spine J 28:544-550

15. Roussouly P, Gollogly S, Berthonnaud E, Dimnet J (2005) Classification of the normal variation in the sagittal alignment of the human lumbar spine and pelvis in the standing position. Spine (Phila Pa 1976) 30:346-353

16. Homans JF, Schlösser TPC, Pasha S et al (2021) Variations in the sagittal spinal profile precede the development of scoliosis: a pilot study of a new approach. Spine J 21:638-641

17. Kouwenhoven J-WM, Smit TH, van der Veen AJ et al (2007) Effects of dorsal versus ventral shear loads on the rotational stability of the thoracic spine: a biomechanical porcine and human cadaveric study. Spine (Phila Pa 1976) 32:2545-2550

18. Homminga J, Lehr AM, Meijer GJM et al (2013) Posteriorly directed shear loads and disc degeneration affect the torsional stiffness of spinal motion segments: a biomechanical modeling study. Spine (Phila Pa 1976) 38:1313-1319

19. Hong J-Y, Suh S-W, Modi HN et al (2010) Correlation of Pelvic Orientation With Adult Scoliosis. J Spinal Disord Tech 23:461-466

20. Wang H, Ma L, Yang DL et al (2015) Radiological analysis of degenerative lumbar scoliosis in relation to pelvic incidence. Int J Clin Exp Med 8:22345-22351

21. Sun XY, Kong C, Zhang TT et al (2019) Correlation between multifidus muscle atrophy, spinopelvic parameters, and severity of deformity in patients with adult degenerative scoliosis: The parallelogram effect of LMA on the diagonal through the apical vertebra. J Orthop Surg Res 14:1-10

22. O'Brien MF, Kulklo TR, Blanke KM, Lenke LG (2008) Radiographic Measurement Manual. Spinal deformity study group (SDSG). Medtronic Sofamor Danek USA, Inc

23. Chow S-C, Shao J, Wang H (2008) Sample Size Calculations in Clinical Research, 2nd edn. Chapman \& Hall/CRC Biostatistics Series, Boca Raton

24. McAviney J, Roberts C, Sullivan B et al (2020) The prevalence of adult de novo scoliosis: A systematic review and meta-analysis. Eur Spine J 29:2960-2969
25. Kelly A, Younus A, Lekgwara P (2020) Adult degenerative scoliosis - A literature review. Interdiscip Neurosurg 20:100661

26. Grivas TB, Vasiliadis E, Malakasis M et al (2006) Intervertebral disc biomechanics in the pathogenesis of idiopathic scoliosis. Stud Health Technol Inform 123:80-83

27. de Vries AAB, Mullender MG, Pluymakers WJ et al (2010) Spinal decompensation in degenerative lumbar scoliosis. Eur Spine J 19:1540-1544

28. Aebi M (2005) The adult scoliosis. Eur Spine J 14:925-948

29. Kettler A, Rohlmann F, Ring C et al (2011) Do early stages of lumbar intervertebral disc degeneration really cause instability? Evaluation of an in vitro database. Eur Spine J 20:578-584

30. Schlösser TPC, Vincken KL, Rogers K et al (2015) Natural sagittal spino-pelvic alignment in boys and girls before, at and after the adolescent growth spurt. Eur Spine J 24:1158-1167

31. Schlösser TPC, Janssen MMA, Vrtovec T et al (2014) Evolution of the ischio-iliac lordosis during natural growth and its relation with the pelvic incidence. Eur Spine J 23:1433-1441

32. Mac-Thiong J-M, Berthonnaud É, Dimar JR et al (2004) Sagittal Alignment of the Spine and Pelvis During Growth. Spine (Phila Pa 1976) 29:1642-1647

33. Mac-Thiong J-M, Labelle H, Berthonnaud E et al (2007) Sagittal spinopelvic balance in normal children and adolescents. Eur Spine J 16:227-234

34. Kouwenhoven JW, Vincken KL, Bartels LW, Castelein RM (2006) Analysis of preexistent vertebral rotation in the normal spine. Spine (Phila Pa 1976) 31:1467-1472

35. Taylor JR (1975) Growth of human intervertebral discs and vertebral bodies. J Anat 120:49-68

36. Edelson JG, Nathan H (1988) Stages in the natural history of the vertebral end-plates. Spine (Phila Pa 1976) 13:21-26

37. Uys A, Bernitz H, Pretorius S, Steyn M (2019) Age estimation from anterior cervical vertebral ring apophysis ossification in South Africans. Int J Legal Med 133:1935-1948

38. Bezci SE, Eleswarapu A, Klineberg EO, O'Connell GD (2018) Contribution of facet joints, axial compression, and composition to human lumbar disc torsion mechanics. J Orthop Res $36: 2266-2273$

Publisher's Note Springer Nature remains neutral with regard to jurisdictional claims in published maps and institutional affiliations. 